\title{
EMMY NOETHER AND LINEAR EVOLUTION EQUATIONS
}

\author{
P. G. L. LEACH ${ }^{a, b, *}$ \\ ${ }^{a}$ School of Mathematical Sciences, University of KwaZulu-Natal, Private Bag X54001, Durban 4000, Republic of \\ South Africa \\ ${ }^{b}$ Department of Mathematics and Statistics, University of Cyprus, Lefkosia, Republic of Cyprus \\ * corresponding author: leachp@ukzn.ac.za
}

\begin{abstract}
Noether's Theorem relates the Action Integral of a Lagrangian with symmetries which leave it invariant and the first integrals consequent upon the variational principle and the existence of the symmetries. These each have an equivalent in the Schrödinger Equation corresponding to the Lagrangian and by extension to linear evolution equations in general. The implications of these connections are investigated.
\end{abstract}

KEYwords: Lagrangian, Hamitonian, Schrödinger, Black-Scholes-Merton.

\section{Noether's THEOREM}

In 1918 Emmy Noether [13] presented a theorem in the Festschrift to mark the Diamond Jubilee of the thesis of Felix Klein. Her presentation dealt with a functional of several independent variables. Given our present interest we quote the theorem for one independent variable, one dependent variable and a first-order Lagrangian.

If the Action Integral

$$
A=\int_{t_{0}}^{t_{1}} L(t, x, \dot{x}) \mathrm{d} t
$$

is invariant under the infinitesimal transformation generated by

$$
\Gamma=\tau \partial_{t}+\eta \partial_{x}
$$

then there exists a function $f(t, x)$ such that

$$
\dot{f}=\tau \frac{\partial L}{\partial t}+\eta \frac{\partial L}{\partial x}+(\dot{\eta}-\dot{x} \dot{\tau}) \frac{\partial L}{\partial \dot{x}}+\dot{\tau} L .
$$

In the present context of the functions $\tau$ and $\eta$ are to be considered as depending upon $t$ and $x$ only, but in a more general context they can also depend upon $\dot{x}$.

The function, $f(t, x)$, is the variation induced in $A$ by the variation in the limits in the Action Integral. Thus it is called a boundary term despite the earnest efforts of successors to describe it as a gauge function.

When the variational principle is invoked, it follows that there is a first integral given by

$$
I=f-\left(\tau L+(\eta-\dot{x} \tau) \frac{\partial L}{\partial \dot{x}}\right) .
$$

Over the approximately 90 years since Noether presented her theorem there have been many efforts to misquote it. As a consequence of these various failures to understand the quite clear exposition in Noether's paper various stratagems have been advanced to remedy the perceived deficiencies of the Theorem. Generally speaking these advances were not necessary.

\section{Quantisation}

Around 1835 Hamilton essentially reduced the study of second-order equations to first-order equations by the introduction - indeed in light of Newton's Second Law, reintroduction - of momentum as a variable conjugate to position. The momentum was defined according to

$$
p=\frac{\partial L}{\partial \dot{x}}
$$

and a new function, nowadays called the Hamiltonian, was introduced according to the formula

$$
H=p \dot{x}-L .
$$

Quite a deal of interesting theory was developed and became the substance of Hamiltonian Mechanics. However, our present interest is the observation by Dirac in 1926 of the resemblance of the operators needed for Quantum Mechanics and the canonical variables of Hamiltonian Mechanics and their Poisson Brackets. Thus the Schrödinger Equation could be written as

$$
i \hbar \frac{\partial u}{\partial t}=\hat{H} u
$$

where $\hat{H}$ is the operator obtained by replacing the variables $x$ and $p$ by the operators $\hat{x}$ and $\hat{p}$.

A matter which should be of some interest is the nature of the function $H$, which can be used as an operator in the Schrödinger Equation.

This is not a trivial question for it must be borne in mind that Hamiltonian Mechanics is a reformulation of Lagrangian Mechanics, which is based upon Newtonian Mechanics, and the fundamental object of the last is Newton's Equation of Motion. Whether one applies Hamilton's Equations or the Euler-Lagrange Equation, the end result should be Newton's Equation. It is evident from Dirac's book [4] that he considered the appropriate Hamiltonian to be the energy, and a 


\begin{tabular}{ll}
\hline Symmetry & Boundary Term \\
\hline$\Sigma_{1}=\partial_{t}$ & $f_{1}=0$ \\
\hline$\Sigma_{2}=t \partial_{t}+\frac{1}{2} x \partial_{x}$ & $f_{2}=0$ \\
\hline$\Sigma_{3}=t^{2} \partial_{t}+t x \partial_{x}$ & $f_{3}=\frac{1}{2} x^{2}$ \\
\hline$\Sigma_{4}=\partial_{x}$ & $f_{4}=0$ \\
\hline$\Sigma_{5}=x \partial_{x}$ & $f_{5}=x$ \\
\hline & $f_{6}=1$ \\
\hline
\end{tabular}

TABLE 1. Noether point symmetries.

conserved energy at that. This marks a strong constraint upon the nature of the Hamiltonian and hence the Lagrangian.

As a simple example consider the two Lagrangians for the free particle given by

$$
L_{1}=\frac{1}{2} \dot{x}^{2} \quad \text { and } \quad L_{2}=\frac{1}{\dot{x}} .
$$

Both give rise to the Newtonian Equation

$$
\ddot{x}=0 .
$$

Both have the maximal number of Noether symmetries, five, for the Lagrangian of a one-degree-offreedom system. The former represents the energy.

Why choose one above the other?

If we do the usual trick of setting Plank's constant to unity in the Schrödinger Equation for the free particle obtained by using the Hamiltonian operator representing the energy, the equation is

$$
2 i \frac{\partial u}{\partial t}+\frac{\partial^{2} u}{\partial x^{2}}=0
$$

A calculation of the Lie point symmetries results in

$$
\begin{array}{lrl}
\Gamma_{1} & =\partial_{t}, & \Gamma_{2}=2 t \partial_{t}+x \partial_{x}, \\
\Gamma_{3}=2 t^{2} \partial_{t}+2 t x \partial_{x}+\left(i x^{2}-t\right) u \partial_{u}, \\
\Gamma_{4}=\partial_{x}, & \Gamma_{5}=t \partial_{x}+i x u \partial_{u}, \\
\Gamma_{6}=u \partial_{u}, & \Gamma_{\infty}=f(t, x) \partial_{u},
\end{array}
$$

where the coefficient function in $\Gamma_{\infty}$ represents a solution of the original equation and shall not be of further interest here. The symmetry, $\Gamma_{6}$ is a consequence of the homogeneity in $u$ of the equation. The remaining five symmetries are closely related to the five Noether point symmetries of the Lagrangian

$$
L=\frac{1}{2} \dot{x}^{2} .
$$

The latter with the boundary term are in Table 1 which are not quite the same in that the boundary terms and the coefficient of $u \partial_{u}$ are not identical, but are suggestive of some connection. This connection is made clearer when one writes

$$
\begin{aligned}
\Gamma= & a(t) \partial_{t}+\left(\frac{1}{2} \dot{a}(t)+b(t)\right) \partial_{x} \\
& +\left(\frac{1}{4} i \ddot{a}(t) x^{2}+i \dot{b}(t) x+c(t)\right) u \partial_{u} \text { and } \\
\Sigma= & a(t) \partial_{t}+\left(\frac{1}{2} \dot{a}(t)+b(t)\right) \partial_{x} \\
& \text { with } \quad f(t, x)=\frac{1}{4} \ddot{a}(t) x^{2}+\dot{b}(t) x+c(t),
\end{aligned}
$$

where in the latter case $c(t)=C_{0}$ and in the former case $c(t)=C_{0}-\frac{1}{4} \dot{a}(t)$.

There is a precise connection.

\section{THE HEAT EQUATION}

The Schrödinger Equation for the free particle,

$$
2 i \frac{\partial u}{\partial t}+\frac{\partial^{2} u}{\partial x^{2}}=0
$$

may be rewritten as

$$
\frac{\partial u}{\partial i t / 2}-\frac{\partial^{2} u}{\partial x^{2}}=0
$$

so that the change of variable, $i t / 2 \longrightarrow \tilde{t}$, gives the classical heat equation

$$
\frac{\partial u}{\partial \tilde{t}}-\frac{\partial^{2} u}{\partial x^{2}}=0
$$

Since the transformation is a point transformation, the number of Lie point symmetries is unchanged. Specifically they are

$$
\begin{array}{rlrl}
\Delta_{1} & =\partial_{t}, & \Delta_{2} & =2 t \partial_{t}+x \partial_{x}, \\
\Delta_{3} & =4 t^{2} \partial_{t}+4 t x \partial_{x}-\left(2 t+x^{2}\right) u \partial_{u} \\
\Delta_{4} & =\partial_{x}, & \Delta_{5} & =2 t \partial_{x}-x u \partial_{u}, \\
\Delta_{6} & =u \partial_{u}, & \Delta_{\infty} & =f(t, x) \partial_{u},
\end{array}
$$

where, as has been the case above, $f(t, x)$ is any solution of the heat equation.

The Lie algebra of the symmetries is $\left\{\operatorname{sl}(2, R) \oplus_{s}\right.$ $\left.W_{3}\right\} \oplus_{s} \infty A_{1}$. The symmetries, $\Delta_{1}$ and $\Delta_{2}$, play a similar role to those of the quantal simple harmonic oscillator as creation and annihilation operators. They have been used to generate heat polynomials [8].

\section{The WONDERFUL WORLD OF FINANCE}

Approximately 40 years ago Black and Scholes [1, 2] and, independently, Merton [9-12] developed a model for the pricing of options. At the time it was remarked that the subject under investigation was of no great importance since trading in options was a minor feature of the Financial Markets. A later observation about the applicability of the model to any financial instrument the future of which was uncertain at the present time certainly extended the relevance of the equation proposed. The Black-Scholes-Merton Equation,

$$
u_{t}+\frac{1}{2} \sigma^{2} x^{2} u_{x x}+r x u_{x}-r u=0,
$$

was originally presented by Black and Scholes, but Merton's name is usually added to indicate his contribution to the development of this part of the theory of Financial Mathematics. This model is the precursor of the many evolution of partial differential equations which have been derived in the modelling of various 
financial processes. Basically it has to do with the pricing of options, but anything vaguely connected such as corporate debt is equally grist for its mill.

The symmetry analysis of $(*)$ was firstly undertaken by Gasizov and Ibragimov [5]. After determining the symmetries they obtained from the solution for the initial condition being a delta function which is a typical initial condition for the heat equation! 1 A more typical problem is the solution of (*), the subject of which is known as a terminal condition, ie $u(T, x)=U$ when $t=T$.

The Lie point symmetries of $(*$ are

$$
\begin{aligned}
\Gamma_{1}= & x \partial_{x} \\
\Gamma_{2}= & 2 t x \partial_{x}+\left\{t-\frac{2}{\sigma^{2}}(r t-\log x)\right\} u \partial_{u} \\
\Gamma_{3}= & u \partial_{u} \\
\Gamma_{4}= & \partial_{t} \\
\Gamma_{5}= & 8 t \partial_{t}+4 x \log x \partial_{x} \\
& +\left\{4 t r+\sigma^{2} t+2 \log x+\frac{4 r}{\sigma^{2}}(r t-\log x)\right\} u \partial_{u} \\
\Gamma_{6}= & 8 t^{2} \partial_{t}+8 t x \log x \partial_{x}+\left\{-4 t+4 t^{2} r+\sigma^{2} t^{2}\right. \\
& \left.\quad+4 t \log x+\frac{4}{\sigma^{2}}(r t-\log x)^{2}\right\} u \partial_{u}
\end{aligned}
$$

$\Gamma_{\infty}=f(t, x) \partial_{u}$,

where $\Gamma_{\infty}$ is the infinite subset of solutions to $*$. The algebra of the finite subset is $\operatorname{sl}(2, R) \oplus_{s} W_{3}$, where $W_{3}$ is the three-dimensional Heisenberg-Weyl algebra.

The important thing to note is that the algebra of the symmetries presented above is just that of the classical equation which we have seen is related to the Schrödinger Equation for the free particle and so to the Noether point symmetries of a classical Lagrangian. A question of course could be posed as to the identity of the classical Lagrangian. Equation $(*)$ is not quite in the form of the classical heat equation, for which we could easily make a definite identification of the corresponding Lagrangian. Under the transformations

$$
\begin{aligned}
& x \mapsto \exp [\sigma y], \\
& u(t, x) \mapsto w(t, y) \exp \left[\frac{1}{2}\left(1-\frac{2 r}{\sigma^{2}}\right) y+\frac{1}{8 \sigma^{2}}\left(\sigma^{2}+2 r\right)^{2}\right]
\end{aligned}
$$

we obtain

$$
2 w_{t}+w_{y y}=0 .
$$

One can detect a certain degree of irony in the identification of the most famous equation of Financial Mathematics with the free particle.

The Black-Scholes-Merton Equation is but one of a number of evolution equations to be found in Financial

\footnotetext{
${ }^{1}$ One would hope that this initial condition would not apply in financial matters! Unfortunately there are some instances of financial instability in which such an initial condition is far too accurate a model. Note that the paper 7] with more realistic conditions appeared earlier, but the content of [5] had already been presented at a seminar in the Department of Physics, the University of the Witwatersrand, Johannesburg, in 1996.
}

Mathematics which are rather blessed with an abundance of symmetry. Admittedly not all of them possess the richness of the algebra $\left\{s l(2, R) \oplus_{s} W_{3}\right\} \oplus_{s} \infty A_{1}$, but one can be successful in the resolution of the equation with fewer symmetries. These algebraic properties are not confined to $1+1$ evolution equations. An interesting example is to be found in the model of the pricing of commodities developed by Eduardo Schwartz [3, 6, 14, which was examined from the viewpoint of symmetry in [15]. Schwartz considered models with one, two and three 'spatial' variables. In terms of the type of model that he proposed, the number of dimensions becomes irrelevant since there is sufficient increase in symmetry with the increase in the number of dimensions.

It is rather intriguing that so many of the equations which arise in the Mathematics of Finance should be so richly endowed with symmetry. Naturally the possession of symmetry is an indispensable aid to a ready resolution of a differential equation which may explain the comment of a learned referee that any competent applied mathematician can solve these equations without recourse to the arcane methods of a Norwegian mathematician.

\section{INITIAL/BOUNDARY CONDITIONS}

Thus far we have considered only the differential equations and not those pesky little things which accompany them when one wants to state the equation correctly, i.e. the initial and/or boundary conditions. In the case of Classical Mechanics with a single independent variable, the situation is quite simple. One solves the equation by one means or another and then evaluates the constants of integration using the initial conditions. To be quite honest, I have never thought of applying the methodology to be discussed below to an initial value problem in Classical Mechanics!

When it comes to partial differential equations, the situation is not so simple, for one can have both boundary conditions and initial conditions. Actually in the case of financial problems it is usually a terminal condition rather than an initial condition, but that is merely a matter of one's attitude to the arrow of time.

Typically the problem with options is to determine the price which one should pay now, say, to purchase some stock in the future at a now-determined price.

I suspect that we all have our favourites ways to write the one-parameter elements of the Lie symmetries of a differential equation. Nevertheless it is well that from time to time we should be reminded that the differential equation admits a single symmetry. If we are fortunate, the single symmetry will be a multiparameter symmetry which gives some freedom when it comes to dealing with the dreaded boundary/initial conditions. Consider the case of the Black-ScholesMerton Equation, * , and the terminal condition $u(T, x)=U$ when $t=T$. We need a symmetry which 
is compatible with these conditions. We write the symmetry of $*$ as

$$
\Gamma=\Sigma_{i=1}^{i=6} \alpha_{i} \Gamma_{i}
$$

and apply this general symmetry to the dual terminal conditions. Note that the solution symmetries, the components of the subalgebra $\infty A_{1}$, do not enter into the discussion.

The details of the calculation are irrelevant for our present purpose. Suffice it to say that the six constants in the general form of $\Gamma$ are constrained to give two oneparameter symmetries. In general the algebra of these symmetries is non-Abelian. Fortunately there is a very useful theorem which guarantees the uniqueness of the solution of the equation under these circumstances. Consequently one chooses the simpler symmetry for further calculation.

\section{TANTUm Adesse}

I have concentrated in the end on the applications of symmetry to the equations of Financial Mathematics, but it is quite evident that these considerations apply to evolution equations wherever they arise.

The connection between the Lie point symmetries of these evolution partial differential equations and the Noether point symmetries classical Lagrangians has not been exploited.

It may well be that for $1+1$ evolution equations the degree of exploitation may be limited. However, when it comes to more degrees of freedom - as in the commodities model of Schwartz - there may be some benefit in the investigation of the properties that the classically conserved quantities may have for these evolution equations.

Finally there are the questions of alternate Lagrangians and of evolution equations which are not presumed to be linear.

\section{ACKNOWLEDGEMENTS}

This contribution was prepared while PGLL was enjoying the hospitality of Professor MC Nucci and the Dipartimento di Matematica e Informatica, Università di Perugia. The continuing support of the University of KwaZuluNatal and the National Research Foundation of South Africa is gratefully acknowledged. Any opinions expressed in this talk should not be construed as being those of the latter two institutions.

\section{REFERENCES}

[1] Black Fischer \& Scholes Myron (1972) The valuation of option contracts and a test of market efficiency Journal of Finance 27 399-417.

[2] Black Fischer \& Scholes Myron (1973) The pricing of options and corporate liabilities Journal of Political Economy 81 637-659.

[3] Brennan MJ \& Schwartz ES (1985) Evaluating natural resource investments Journal of Business 58 133-155.

[4] Dirac PAM (1932) The Principles of Quantum Mechanics (Cambridge, at the Clarendon Press).

[5] Gasizov R \& Ibragimov NH (1998) Lie symmetry analysis of differential equations in finance Nonlinear Dynamics 17 387-407.

[6] Gibson R \& Schwartz ES (1990) Stochastic convenience yield and the pricing of oil contingent claims Journal of Finance 45 959-976.

[7] Ibragimov NH \& Wafo Soh C (1997) Solution of the Cauchy problem for the Black-Scholes equation using its symmetries Modern Group Analysis, International Conference at the Sophus Lie Conference Centre Ibragimov NH, Naqvi KR \& Straume E edd (MARS Publishers, Norway).

[8] Leach PGL (2006) Heat polynomials and Lie point symmetries Journal of Mathematical Analysis and Applications 322 288-297.

[9] Merton RC (1969) Lifetime portfolio selection under uncertainty: The continuous-time case Review of Economics and Statistics 51 247-257.

[10] Merton RC (1971) Consumption and Portfolio Rules in a Continuous Time Model Journal of Economic Theory 3,4 373-413.

[11] Merton Robert C (1973) Theory of rational option pricing Bell Journal of Economics and Management Science 4 141-183.

[12] Merton RC (1974) On the pricing of corporate data: the risk structure of interest rates Journal of Finance 29 449-470.

[13] Noether Emmy (1918) Invariante Variationsprobleme Königlich Gesellschaft der Wissenschaften Göttingen Nachrichten Mathematik-physik Klasse 2 235-267.

[14] Schwartz ES (1997) The stochastic behaviour of commodity prices: implications for valuation and hedging the Journal of Finance 52 923-973.

[15] Sophocleous C, Leach PGL \& Andriopoulos K (2008) Algebraic properties of evolution partial differential equations modelling prices of commodities Mathematical Methods in the Applied Sciences 31 679-694. 